\title{
Auditory induction: Reciprocal changes in alternating sounds
}

\author{
RICHARD M. WARREN, JAMES A. BASHFORD, JR., ERIC W. HEALY, \\ and BRADLEY S. BRUBAKER \\ University of Wisconsin-Milwaukee, Milwaukee, Wisconsin
}

\begin{abstract}
When portions of a sound are replaced by a potential masker, the missing fragments may be perceptually restored, resulting in apparent continuity of the interrupted signal. This phenomenon has been examined extensively by using pulsation threshold, auditory induction, and phonemic restoration paradigms in which two sounds, the inducer and the inducee, are alternated (ABABA...), and the conditions required for apparent continuity of the lower amplitude inducee are determined. Previous studies have generally neglected to examine concomitant changes produced in the inducing sound. Results from the present experiments have demonstrated decreases in the loudness of inducers using inducer/inducee pairs consisting of tone/tone and noise/noise, as well as the noise/speech pairs associated with phonemic restorations. Interestingly, reductions in inducer loudness occurred even when the inducee was heard as discontinuous, and these decreases in loudness were accompanied by graded increases in apparent duration of the inducee, contrary to the conventional view of auditory induction as an all-or-none phenomenon. Under some conditions, the reduced loudness of the inducer was coupled with a marked alteration in its timbre. Especially profound changes in the inducer quality occurred when the alternating stimuli were tones having the same frequency and differing only in intensity-it seems that following subtraction of components corresponding to the inducee, an anomalous auditory residue remained that did not correspond to the representation of a tone.
\end{abstract}

Under the noisy conditions encountered in everyday life, signals of interest are often masked by extraneous sounds. However, listeners are capable of restoring missing fragments of signals: If a brief interrupting sound obliterates a portion of the signal, listeners restore the missing segment on the basis of the intact portions and cannot distinguish the perceptually synthesized segment from those segments actually present.

In most laboratory studies of this "auditory induction" of missing sounds, a fainter sound, the "inducee," is alternated in a regular fashion with a sound of higher amplitude, the "inducer," in the fashion ABABA... . These studies have generally concentrated on apparent continuity of the inducee and have neglected to examine possible concomitant changes in the inducer. Although it has been suggested that a portion of the neural response to the inducer is subtracted and used for the perceptual synthesis of the inducee (Warren, Obusek, \& Ackroff, 1972), direct experimental data supporting this hypothesis is lacking. The series of experiments described here examine consequences of the hypothesis that restoration is based on a reciprocal interaction involving the allocation of a portion of the auditory input produced by the inducer over

This study was supported by a grant from the National Institute on Deafness and Other Communication Disorders (DC00208). The valuable contributions of Keri R. Riener and Makio Kashino are gratefully acknowledged. Correspondence should be addressed to R. M. Warren, Department of Psychology, Garland Hall, University of WisconsinMilwaukee, P.O. Box 413, Milwaukee, WI 53201. to the inducee. These experiments employed stimuli that consisted of tones, noises, and speech.

Experiment 1 was designed to measure any changes occurring in the loudness of a sinusoidal inducer tone $(1000 \mathrm{~Hz}$ at $70 \mathrm{~dB})$ when alternated with sinusoidal inducees having various frequencies and amplitudes. In keeping with the neural reallocation hypothesis, there was a reciprocal interaction of the tones, and the loudness of the inducer was decreased when the inducee was restored to apparent continuity. However, reduction of the loudness of the inducer also occurred (to a lesser extent) when the inducee appeared to be discontinuous. This observation appears to conflict with the assumption made in studies reported by Houtgast (1972), Warren et al. (1972), and others that restoration is an all-or-none phenomenon, as is indeed the case when it is measured as the continuity/discontinuity transition or "pulsation threshold" of the inducee (see Warren, 1984, for a review of this literature).

The loudness reduction of the inducer in the absence of continuity suggested that changes in the inducee might also be occurring below the continuity threshold, perhaps involving an increase in the apparent duration of the inducee that was insufficient to completely bridge the gap separating successive statements. Experiment 2 was undertaken to determine the apparent duration of the inducee under the same conditions of inducer-inducee alternation employed in Experiment 1. The results obtained in Experiment 2 supported the hypothesis that the extent of inducer-inducee interaction represents a continuum having 
complete gap closure and continuity of the inducee as an endpoint.

One of the conditions employed in both Experiments 1 and 2 involved alternating sinusoidal tones having an identical frequency but different amplitudes. The resulting "homophonic induction" represents an interesting type of interaction. As the inducee level was raised from threshold while the level of the homophonic inducer remained fixed, the inducer not only seemed to become progressively fainter, it also became less tonal and was heard as a novel harsh sound. When the inducee reached a level 1-3 dB below that of the inducer, the percept was often difficult to describe, and some listeners reported that the stimulus seemed to exhibit aspects of both auditory induction and amplitude modulation of a single pure tone.

Experiment 3 expanded the investigation of changes in a homophonic inducer to an aperiodic signal-broadband noise. This homophonic stimulus pair consisted of two broadband noises having identical long-term spectral profiles and differing only in amplitude (a 70-dB inducer alternating with an inducee of either 68 or $66 \mathrm{~dB}$ ). The interaction of the homophonic noise pairs in Experiment 3 differed from that observed with homophonic tone pairs in Experiment 1, suggesting differences in the neural coding of amplitude for the two types of sound.

Experiment 4 deals with the relation of the "phonemic restoration" of speech interrupted by noise to the restoration of fragments of nonverbal sounds. It has been suggested that phonemic restorations represent a verbal form of auditory induction that is subject to the same basic rules as those governing the restoration of nonverbal sounds, but which also involves the application of linguistic skills that enable listeners to employ intact portions of the signals to identify and synthesize missing segments (Warren et al., 1972; Warren \& Sherman, 1974). In keeping with this suggestion, it has been reported that the spectral relations of inducer and inducee required for restoration of speech sounds matched those required for nonverbal restoration (Bashford, Riener, \& Warren, 1992; Bashford \& Warren, 1979, 1987). Although Bregman (1990) has agreed that restoration of tones involves an allocation of some of the inducer's neural response to the inducee, he has hypothesized that phonemic restorations are fundamentally different, representing a "schemadriven stream segregation" in which no such reallocation occurs (p. 372). To determine directly whether or not the reallocation of inducer input occurs with phonemic restorations, Experiment 4 alternated narrowband speech with narrowband noise of various center frequencies. As we shall see, a decrease in loudness of the narrowband noises serving as inducers did occur, and the extent of reduction exhibited the spectral dependency found for tonal induction in Experiment 1.

\section{GENERAL METHOD}

Subjects. The 4 subjects who participated in Experiments 1A, 3 , and 4 were laboratory personnel with prior experience as listeners in psychophysical studies. Three of these listeners also participated in Experiments 1B and 2. All listeners received several hours of initial training in matching the apparent levels of tones and of noise stimuli presented in isolation prior to their participation in the formal experiments involving alternating sounds.

Stimuli. In each experiment, the induction stimuli consisted of two sounds that were presented in an alternating fashion (ABABA...). The on-time and off-time for each sound were equal and were $200 \mathrm{msec}$ for the alternating tones presented in Experiments 1 and 2 and also for the alternating levels of broadband white noise presented in Experiment 3. In Experiment 4, which presented narrowband speech alternating with various narrow bands of noise, on/off times were $125 \mathrm{msec}$. To reduce switching transients, signals were gated on and off with a 10 -msec raised cosine ramp using Coulbourn Model S84-04 electronic switches triggered by Grason Stadler 1200 Series programming equipment. The outputs of the electronic switches were amplified with a Neotek Series I audio mixer and delivered diotically through Sennheiser HD 230 circumaural headphones. The inducer in each experiment was presented at an SPL of $70 \mathrm{~dB}$ (A-scale weighting was used throughout this study). The inducee signals were presented either at the same SPL as the inducer (Experiment 4) or at lower SPLs (Experiments 1-3).

Signal levels were measured with a Brüel and Kjær Model 2230 sound-level meter fitted with a flat-plate coupler and operating in its slow-average mode. The waveforms and spectra of all signals-as transduced by the Sennheiser headphones-were examined with a Hewlett-Packard Model 3561A signal analyzer.

\section{EXPERIMENT 1}

\section{Method}

Stimuli and Procedure. The inducer employed in Experiment 1 was always a $70-\mathrm{dB}, 1-\mathrm{kHz}$ sinusoidal tone that alternated with a lower amplitude sinusoidal tone serving as inducee. In Experiment $1 \mathrm{~A}$, the inducees were $66-\mathrm{dB}$ tones that either matched the inducer in frequency or differed by $\pm 1,2,6$, or 10 semitones (i.e., inducee frequencies of $561,707,891,944,1000,1059,1122,1414$, and $1782 \mathrm{~Hz}$ ). Experiment $1 \mathrm{~B}$ employed three inducee amplitudes $(63,60$, and $55 \mathrm{~dB})$, with each level presented at only three frequencies $(1 \mathrm{kHz}$, one semitone above $1 \mathrm{kHz}$, and one semitone below $1 \mathrm{kHz}$ ).

The inducer tone was produced by a Wavetek Model $171 \mathrm{syn}$ thesizer and delivered at $70 \pm 0.1 \mathrm{~dB}$ at each headphone. The comparison tone employed for loudness matching was produced by a Hewlett-Packard 3325A synthesizer under the control of a HewlettPackard Model HP-85 computer. The inducee tones were produced by a two-channel synthesizer (Hewlett-Packard 3326) that also was controlled by the HP-85 computer. The computer varied the frequency of the inducee and also adjusted separately the amplitudes of the outputs from the synthesizer's two channels in order to compensate for differences in earphone response: A computer program calibrated separately for each headphone permitted the matching of SPL to within $\pm 0.1 \mathrm{~dB}$ at each ear for all inducee frequencies. This precluded differences in lateralization of the inducer and the inducee. Phase relations between inducer and inducee at the initiation of the 10 -msec rise/fall period were randomly determined. ${ }^{1}$

Each experimental trial began with a 3.4-sec induction interval, during which the listener heard nine statements of the inducee (A) alternating with eight statements of the inducer $(B)$ : The sequence of tones began and ended with bursts of the inducee (ABAB ...A). As shown in Figure 1, this induction interval was followed by an 800-msec silent pause and then a 1.4-sec comparison interval, during which the listener heard four bursts of a sinusoid (200 msec on/off times) that matched the duration and $1-\mathrm{kHz}$ frequency of the inducer (B_B_B_B B $_{-}$.

Inducees were presented at a particular amplitude $(66 \mathrm{~dB}$ in Experiment $1 \mathrm{~A} ; 63,60$, and $55 \mathrm{~dB}$ in Experiment $1 \mathrm{~B}$ ), and the listeners 


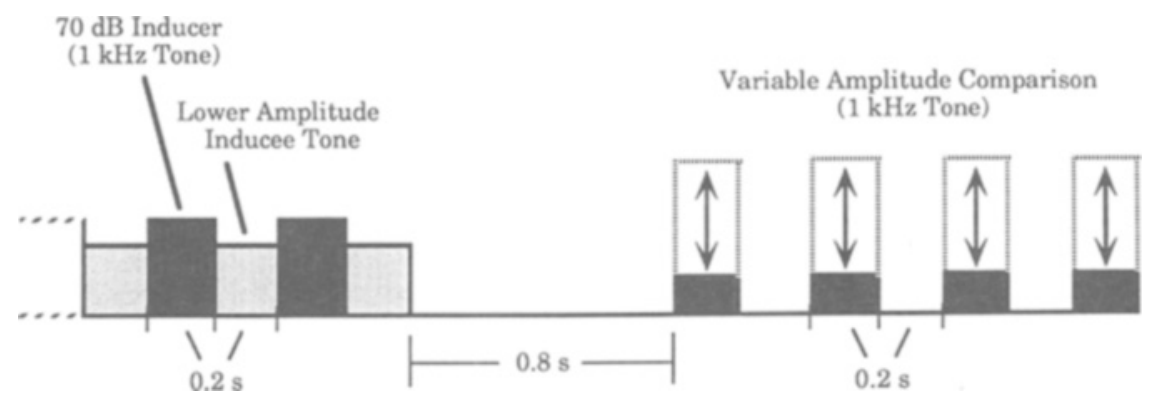

Figure 1. Stimuli employed for measuring the loudness reduction of the $1-\mathrm{kHz}$ inducer during auditory induction. Inducee frequencies ranged from 10 semitones below the inducer $(561 \mathrm{~Hz})$ to 10 semitones above the inducer $(1782 \mathrm{~Hz})$.

participated in four blocks of judgments, with the various inducee frequencies used with that amplitude appearing once in each block. The order of presentation within blocks for inducees was pseudorandom, with the restriction that no frequency recurred in succession across blocks. The listeners judged the apparent level of the inducer by using a modified method-of-limits procedure. By depressing a button, they activated programming equipment that presented them with individual two-interval forced-choice trials, which, as described above, consisted of a 3.4-sec induction interval, an 800msec silent pause, and then four bursts of the comparison tone that matched the frequency of the inducer. On the beginning trial of an ascending series of judgments, the comparison tone was presented at a low sound pressure level-varying randomly from 53 to $58 \mathrm{~dB}$-and increased regularly in steps of $2 \mathrm{~dB}$ for successive trials until the listener judged the comparison tone to be louder than the 70-dB inducer. For a descending series of trials, the comparison tone started at a level varied randomly from 79 to $84 \mathrm{~dB}$ and then decreased in 2-dB steps until the listener judged the comparison to be fainter than the inducer. For each presentation of a given inducee frequency, the listener was presented alternately with ascending and descending series of trials for a total of 10 series for each of the nine stimulus pairs employed in Experiment $1 \mathrm{~A}$ and the nine stimulus pairs employed in Experiment 1B.

\section{Results and Discussion}

Figure 2 presents the results for loudness reduction obtained in Experiment 1A, in which the 70- $\mathrm{dB}$ inducer tone alternated with a $66-\mathrm{dB}$ inducee tone at the various frequencies. The frequency differences between the inducees and the inducer are given in semitones (i.e., 1/12octave steps), and these separations ranged from -10 to +10 semitones. Figure 2 indicates that the greatest loudness reduction for the inducer occurred under homophonic conditions ( 0 -semitone separation), with a decrease of more than $5 \mathrm{~dB}$ in apparent level. The decrease in loudness produced by the 66- $\mathrm{dB}$ inducees appears to be an asymmetrical function of frequency separation. There was no reduction in loudness with -10 and -6 semitone inducees, with the inducer judged to be as loud as a $70-\mathrm{dB}$

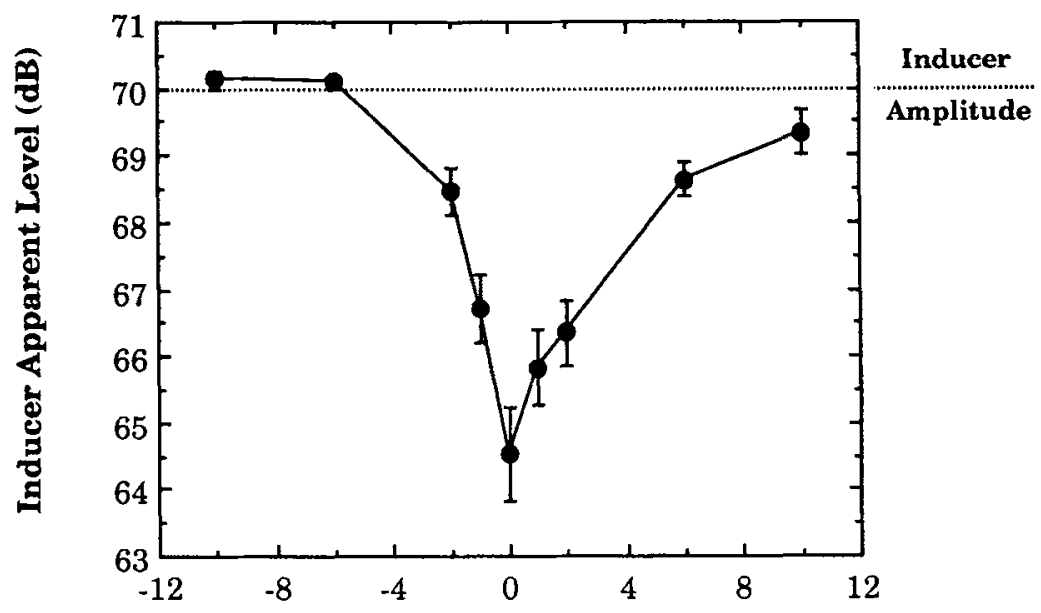

Inducee Frequency Relative to Inducer (semitones)

Figure 2. Auditory induction: Changes produced in the apparent level (loudness) of a 70-dB, 1-kHz inducer alternating with 66-dB inducees of various frequencies. Inducee frequencies are given as the difference in semitones from the inducer frequency (each semitone is $1 / 12$ octave). Plotted values are means and standard errors for 4 listeners. 
comparison stimulus. The loudness of the inducer then dropped steeply as the frequency of the 66- $\mathrm{dB}$ inducee increased up to the homophonic value of $1000 \mathrm{~Hz}$ and then changed more gradually as the frequency of the inducee was raised above that value. This is consistent with the asymmetrical upward spread of neural activity expected for the 70-dB inducer. For those inducees with frequencies higher than that of the inducer, this asymmetry provides a greater tonotopic overlap of excitation produced by the alternating stimuli. It is this overlap that provides the opportunity for the reallocation of a portion of the inducer's auditory input.

These observations were supported by statistical analyses. The listeners' mean matches obtained for the various 66- $\mathrm{dB}$ inducees were subjected to a treatments $\times$ subjects analysis of variance (ANOVA) that yielded a significant effect of inducee frequency $[F(8,24)=27.27, p<$ $.0001]$. Evidence of the predicted asymmetry in the effects of heterophonic inducees was obtained in subsequent, single-degrees-of-freedom $(d f)$ contrasts (Neter \& Wasserman, 1974), which compared the mean of the four lowest inducee conditions (i.e., $-10,-6,-2$, and -1 semitone inducees) with the mean of the four highest inducee conditions $(+10,+6,+2$, and +1 semitone inducees): A greater loudness decrease was produced by the higher frequency inducees $[F(1,24)=23.38, p<$ $.0001]$. Also as predicted, the mean for the 0-semitone inducee condition was significantly lower $[F(1,24)=$ 13.66, $p<.002]$ than the combined mean for the +1 and -1 semitone inducees, indicating that reallocation of the inducer's input was greatest in the homophonic induction condition. $^{2}$

Results obtained in Experiment 1B for inducees at 63, 60 , and $55 \mathrm{~dB}$ are shown for $-1,0$, and +1 semitone separations in Figure 3. An examination of the figure indicates that, under homophonic conditions, there was a monotonic decrease in the apparent level of the inducer as the inducee increased from 55 to $63 \mathrm{~dB}$. This is in keeping with induction theory, which requires a smaller loudness reduction of the inducer as reduced proportions of the auditory input are reallocated to produce the apparent continuity of the inducee. These observations are also supported by statistical analyses. Listeners' mean matches were subjected to a treatments $\times$ treatments $\times$ subjects ANOVA that yielded a significant main effect for inducee amplitude $[F(2,4)=15.83, p<.02]$. Subsequent, single-df comparisons of the combined means for each inducee amplitude indicated that the $63-\mathrm{dB}$ inducees produced greater reduction of inducer loudness than did the 60 -dB inducees $[F(1,8)=9.81, p<.02]$ which in turn produced greater reduction than did the $55-\mathrm{dB}$ inducees $[F(1,8)=24.21, p<.002]$.

Under some homophonic conditions, the listeners reported that the inducer not only dropped in loudness but also no longer sounded like a tone. At low levels of the inducee, continuity occurred with little change in the perceptual quality of the inducer. As the inducee level was increased, the homophonic inducer sounded progressively less like a pure tone, and, at the inducer/inducee ratio of $70 / 66 \mathrm{~dB}$, the inducer often was heard as a harsh, jangling sound. Perhaps the residue remaining after reallocation consists only of that portion of the inducer's auditory representation that corresponds to an increase in stimulus amplitude above that of the inducee. The coding of an increase in amplitude at the level of the auditory nerve is quite complex-it includes an increase in firing rate, a crossing of the threshold for less sensitive fibers having a characteristic frequency corresponding to

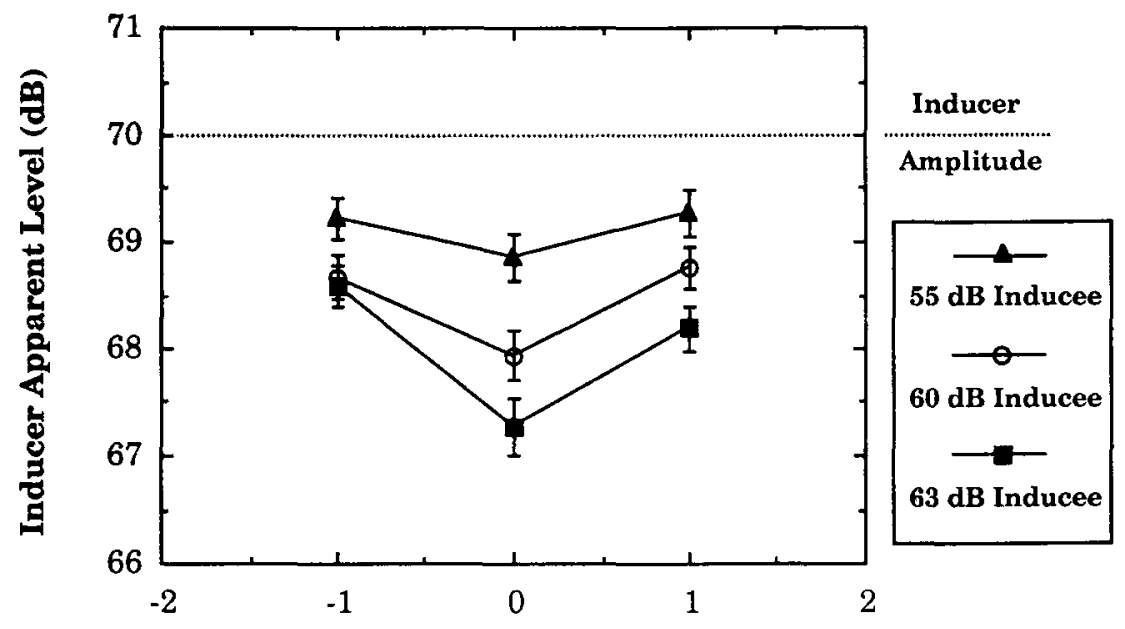

Inducee Frequency Relative to Inducer (semitones)

Figure 3. Auditory induction: Changes produced in apparent level (loudness) of a 70-dB, $1-\mathrm{kHz}$ inducer alternating with inducees of various frequencies and amplitudes. Inducee frequencies are given as the difference in semitones from the inducer frequency (each semitone is $1 / 12$ octave). Plotted values are means and standard errors for 3 listeners. 

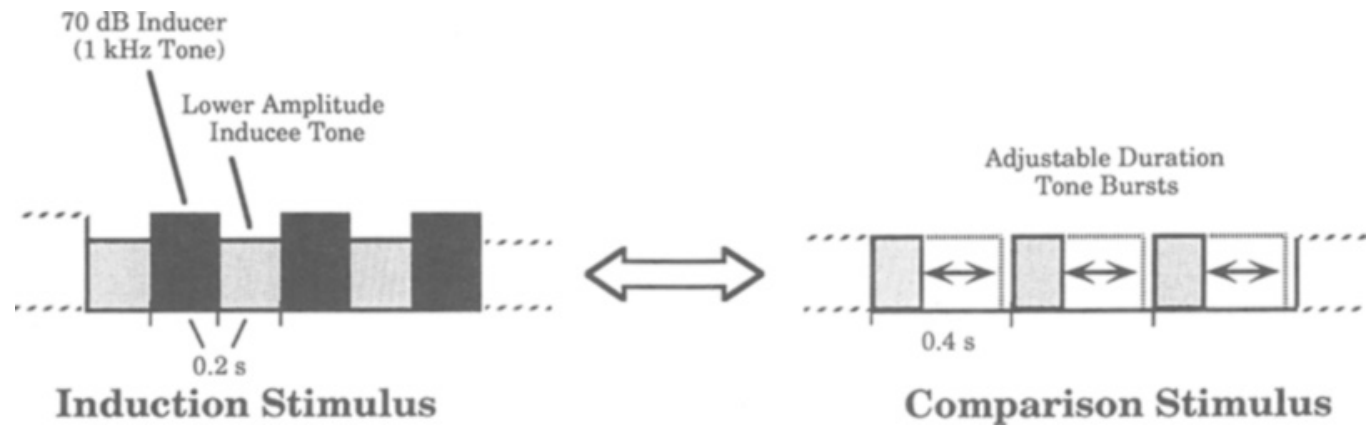

Figure 4. Stimuli employed for measuring the apparent duration of the inducee during auditory induction. Inducee frequencies ranged from 10 semitones below the inducer $(561 \mathrm{~Hz})$ to 10 semitones above the inducer $(1782 \mathrm{~Hz})$.

that of the stimulus, the spread of excitation to fibers differing in characteristic frequency, and complex changes associated with phase locking (for reviews, see Javel, 1986; Smith, 1988). The manner in which these changes are employed for the perceptual representation of a stimulus at a particular level remains to be determined. But however they are used, it would seem that removal of input representing a lower stimulus level leaves an anomalous residue that not only is reduced in loudness but also no longer sounds like a sinusoidal tone.

There is another quite interesting aspect of the loudness reduction shown in Figure 2. Continuity was heard only for homophonic and +1 semitone conditions-for all other frequency separations, the inducee was above the pulsation threshold (consistent with results reported by Warren et al., 1972) and was heard as discontinuous by all listeners. Why, then, is the loudness of the inducer decreased for $-2,-1,+2,+6$, and +10 semitones if there is no inducer/inducee interaction resulting in illusory continuity?

One possible explanation is that the upper limit of continuity (or pulsation threshold) does not represent the point at which the reallocation of neural input to the inducee ceases. Perhaps perceptual transfer of inducer activity can serve to increase the apparent duration of the inducee, but not to an extent sufficient to bridge completely the 200-msec gaps separating successive statements. Evidence that such an incomplete bridging can occur has been reported previously under rather different conditions by Wrightson and Warren (1981), who found that an illusory lengthening of a $1,000-\mathrm{msec}, 1-\mathrm{kHz}$ tone occurred when it was alternated with a louder 500 -msec noise. Encouraged by this earlier study, we undertook Experiment 2 to measure the apparent duration of the inducee under the same conditions that produced the loudness reduction of the inducer measured in Experiment 1A.

\section{EXPERIMENT 2}

\section{Method}

Stimuli. As in Experiment $1 \mathrm{~A}$, the inducer was a $70-\mathrm{dB}, 1-\mathrm{kHz}$ sinusoid presented in alternation (200-msec half-cycle) with 66 -dB sinusoidal inducees having the same nine frequencies employed in that experiment.
Procedure. The method of adjustment was used to obtain estimates of inducee apparent durations. Listeners were provided with a control button that allowed them to switch at will from the standard stimulus, consisting of the alternating inducee (A) and inducer (B) tones (ABABA ...), to a comparison stimulus in which the inducer tone bursts were removed and replaced by silent gaps (A_A_A ...). The remaining comparison tone bursts matched the inducee in frequency and SPL but could be varied in duration, as shown in Figure 4. By adjusting the dial of a function generator (Wavetek Model 135), the listener could vary the comparison burst on-time from a minimum value of $150 \mathrm{msec}$ ( $50 \mathrm{msec}$ shorter than the actual duration of the inducee bursts in the induction stimulus) to a maximum value of $400 \mathrm{msec}-$ with the latter duration closing the silent gaps entirely and presenting a continuous tone to the listener. Programming equipment was arranged so that the onset of the comparison tone occurred every $400 \mathrm{msec}$ at the same time that the onset of the inducee would occur if the standard stimulus were continuing. Thus, there was no disruption of rhythm when the listeners switched from the induction stimulus to the comparison stimulus.

In each session of this experiment, before the listeners provided duration matches for the inducee when it alternated with the inducer, they first made duration matches with the inducer turned off. This provided baseline duration matches in the absence of induction. After making these baseline adjustments, the listeners provided duration matches with the $1-\mathrm{kHz}$ inducer alternating with the inducee. The order of presentation for the nine inducee frequencies was randomized for each session and listener. The listeners participated in a total of 10 sessions, yielding 10 experimental and 10 baseline duration matches for each of the inducees.

\section{Results and Discussion}

The group mean matches for inducee apparent duration are shown in Figure 5. It appears that under baseline conditions (i.e., in the absence of the inducer), the inducee duration could be matched quite accurately. However, when the inducer was present, the apparent duration of the inducee varied from a veridical match of $200 \mathrm{msec}$ to full continuity at $400 \mathrm{msec}$. Thus, the illusory lengthening of the inducee is not an all-or-none phenomenon with an abrupt change occurring at the pulsation threshold, but rather consists of a continuum of values with complete closing of the gap separating successive statements as the upper limit. These observations are supported by statistical analyses. The three listeners' mean matches 


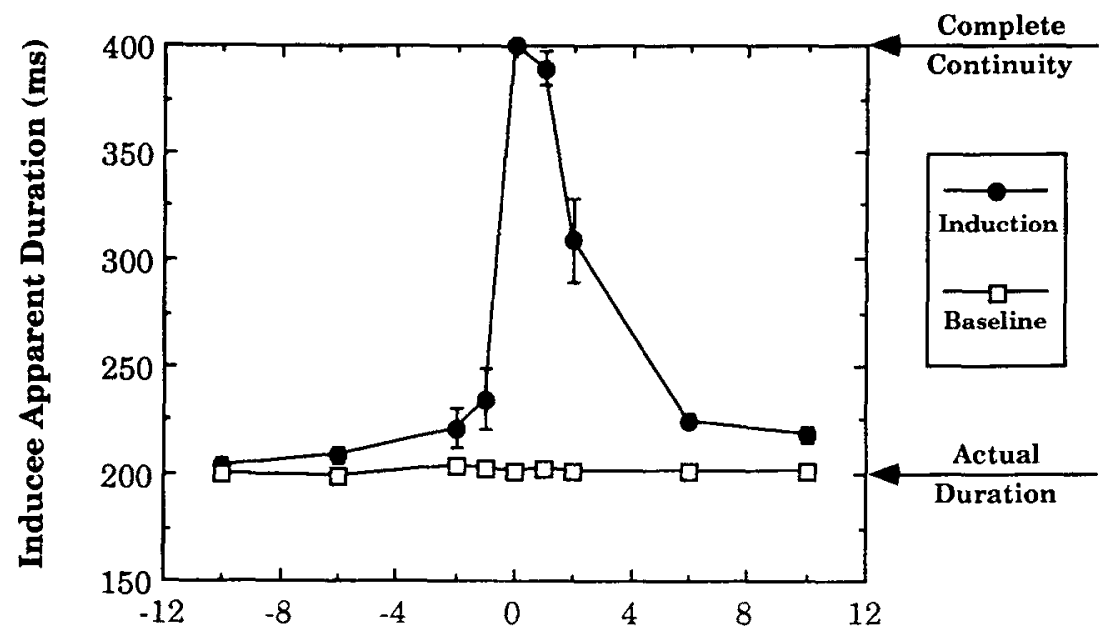

Inducee Frequency Relative to Inducer (semitones)

Figure 5. Auditory induction: Changes produced in the apparent duration of 66-dB sinusoidal inducees of various frequencies alternating with a 70-dB, 1-kHz inducer. The baseline judgments represent the apparent duration of the inducees in the absence of the inducer. Plotted values are means and standard errors for 3 listeners. Inducee frequencies are given as the difference in semitones from the inducer frequency (each semitone is $1 / 12$ octave).

(based on 10 judgments in each condition) were subjected to a treatments $\times$ treatments $\times$ subjects ANOVA, which yielded significant main effects of inducee frequency $[F(8,16)=69.43, p<.0001]$ and inducer condition (i.e., the baseline condition with inducer absent vs. the induction condition with inducer present) $[F(1,2)=135.88, p<$ $.01]$. There was also a significant interaction $[F(8,16)=$ $108.02, p<.0001]$. Subsequent simple-effects analysis of the interaction revealed that the effect of inducee frequency was significant in the induction condition $[F(8,16)$ $=86.74, p<.001]$ but not in the baseline condition $[F(8,16)=0.82, p>.50]$. Subsequent single- $d f$ contrasts indicated that the apparent duration of the inducee did not differ between the baseline and induction conditions for the -10 semitone inducee $[F(1,16)=0.26, p>.60]$ or the -6 semitone inducee $[F(1,16)=1.44, p>.20]$, but did differ at all other inducee frequencies $[F(1,16) \geq$ $5.81, p<.03$ or better].

Figure 6 is based on the data shown in Figures 2 and 5 and shows that changes in judgments of inducee duration as a function of frequency are accompanied by changes in judgments of inducer amplitude. That is, the longer the apparent duration of the inducee, the greater the loudness reduction for the inducer. It is suggested that auditory induction involves a reciprocal interaction of inducer and inducee in which a portion of the neural stimulation produced by the inducer is used for increasing the apparent duration of the inducee, which can extend up to the limiting value of full continuity.

\section{EXPERIMENT 3}

When two noises with identical long-term spectra but different amplitudes are alternated, the lower level is heard as continuous, along with the pulsed addition of the other sound (Warren et al., 1972). Informal observations have indicated that changes in both the timbre and loudness of the inducer are less for homophonic noises than for homophonic tones. In addition, with differences of $3 \mathrm{~dB}$ or less in the levels of alternating homophonic tone pairs, some listeners reported the perception of amplitude modulation mixed with auditory induction. However, for homophonic noise pairs, all listeners reported only auditory induction with level differences of $1 \mathrm{~dB}$ or even less. For level differences below $3 \mathrm{~dB}$, the higher amplitude inducer appeared as a pulsed noise that was clearly fainter than the continuous sound. Experiment 3 was undertaken to obtain quantitative measures of this loudness reduction.

\section{Method}

Stimuli. All stimuli were derived from Gaussian noise produced by a Brüel \& Kjær Model 1405 noise generator and bandpass filtered from $100 \mathrm{~Hz}$ to $8 \mathrm{kHz}$ with a Wavetek Model 852 filter $(48 \mathrm{~dB} / \mathrm{oc}-$ tave slopes). The level of the higher amplitude noise serving as inducer was fixed at $70 \mathrm{~dB}$. The lower level noise serving as the inducee was presented at 66 and $68 \mathrm{~dB}$.

As in Experiment 1, the initial stimulus in each trial consisted of eight statements of the inducer alternating with nine statements of the inducee, with the sequence beginning and ending with bursts of the inducee. This 3.4-sec induction interval was followed by an 800 -msec silent pause and then a 1.4-sec interval that contained four bursts of a comparison noise alternating with silence $(200-\mathrm{msec}$ on/off time). As with the tonal stimuli in Experiment 1, the amplitude of the comparison noise bursts in this study was varied across trials in accord with the method-of-limits procedure described previously.

The level of the comparison noise employed for matching the loudness of the inducer was controlled by a Coulbourn 577-05 voltagecontrolled amplifier. During the experiment, the output level of that amplifier was varied in 2-dB steps across trials by the HewlettPackard HP-85 computer. 


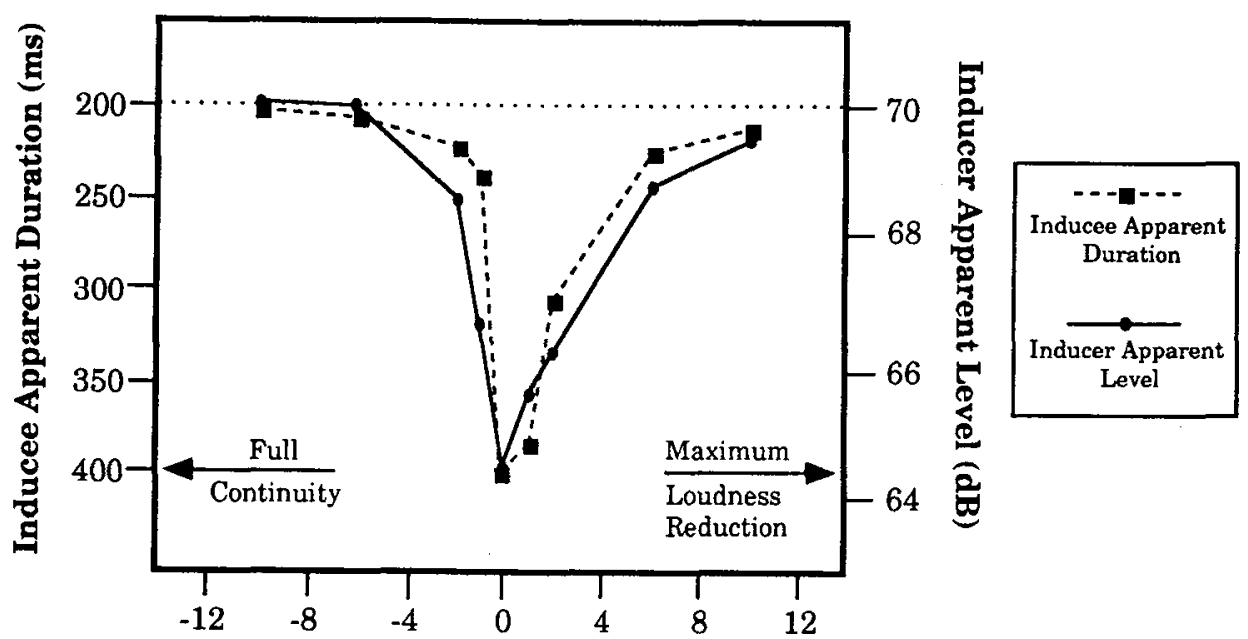

Inducee Frequency Relative to Inducer (semitones)

Figure 6. Auditory induction: Relation of changes in apparent inducee duration, and changes in apparent inducer amplitude. The inducer is a 70-dB, 1-kHz tone and the inducees are 66-dB tones having various frequencies expressed as semitone separation from the inducer frequency (each semitone is $1 / 12$ octave). The horizontal dotted line represents the actual value of inducee duration and the actual value of inducer amplitude. Data are standardized so that the maximum reduction in apparent inducer amplitude (5.5 dB) matches the ceiling value for inducee apparent duration (full continuity).

Procedure. The procedure in this experiment was similar to that used in Experiment 1, except that broadband noise was used instead of tones. As in Experiment 1, for each experimental condition, listeners provided 4 blocks of judgments, with 5 ascending and 5 descending method-of-limits series presented alternately in each block. Thus, 40 series of judgments of the inducer's apparent level were obtained with the inducee noise level fixed at $68 \mathrm{~dB}$, followed by another 40 series of trials obtained with the inducee at $66 \mathrm{~dB}$. In addition, each block of experimental judgments was preceded by a baseline block in which the inducer was present, but the inducee noise bursts were replaced by silence. As with the experimental blocks, each baseline block consisted of 5 paired ascending and descending series of loudness matches.

\section{Results and Discussion}

Figure 7 shows that judgments of the inducer level were accurate when the inducee was replaced by a silent gap (the baseline condition). The agreement of matching judgments with the long-term root-mean-square (rms) amplitude averages of the stochastic signals occurred despite the random fluctuations in amplitude of the individual noise bursts. When the $70-\mathrm{dB}$ inducer was alternated with a 66- $\mathrm{dB}$ inducee, a reduction of about $4 \mathrm{~dB}$ in the apparent level of the inducer took place $[F(1,6)=24.63, p<$ .003 ], so that the two levels heard (the continuous and the pulsed noises) were approximately equal in apparent magnitude. When the homophonic noises differed by $2 \mathrm{~dB}$ $(68 \mathrm{~dB}$ alternated with $70 \mathrm{~dB})$, the reduction in the apparent level of the inducer was approximately $7 \mathrm{~dB}$, a value significantly greater than that observed with the 66-dB inducee $[F(1,6)=13.58, p<.01]$. The greater reduction in the loudness of the inducer when the inducee was closer in amplitude is in agreement both with the observations involving alternating tones in Experiment 1 and with auditory induction theory requiring transfer of a higher pro- portion of auditory input for the perceptual lengthening of the inducee.

\section{EXPERIMENT 4}

Warren et al. (1972) suggested that phonemic restoration of speech is a verbal type of auditory induction subject to the rules governing restoration of nonverbal sounds. A series of studies has been undertaken to test the validity of this suggestion. These studies have found that, in keeping with the spectral requirements for nonverbal auditory induction, the greatest increase in apparent continuity of narrowband speech passages interrupted periodically by narrowband noise occurred when the center frequencies of the speech and noise bands were the same (Bashford \& Warren, 1979, 1987), and also that the intelligibility of narrowband sentences was greatest when the interrupting noise bands matched the center frequency of the speech (Bashford et al., 1992).

Bregman (1990) has suggested that phonemic restoration represents a "schema-driven stream segregation" that is fundamentally different from the auditory induction of nonverbal sounds such as tones and noises. He hypothesized that no reallocation of auditory input from extraneous noise to speech occurs when speech is restored (although he believes that such reallocation does occur with nonverbal induction). Since all of the prior studies dealing with phonemic restorations were concerned with changes involving the speech signal and not the noise, Repp (1992) attempted to determine whether or not perceptual changes occurred in the noise. He replaced the phoneme $/ \mathrm{s} /$ in a word with a noise having a different spectral profile from that of the hiss corresponding to the pho- 


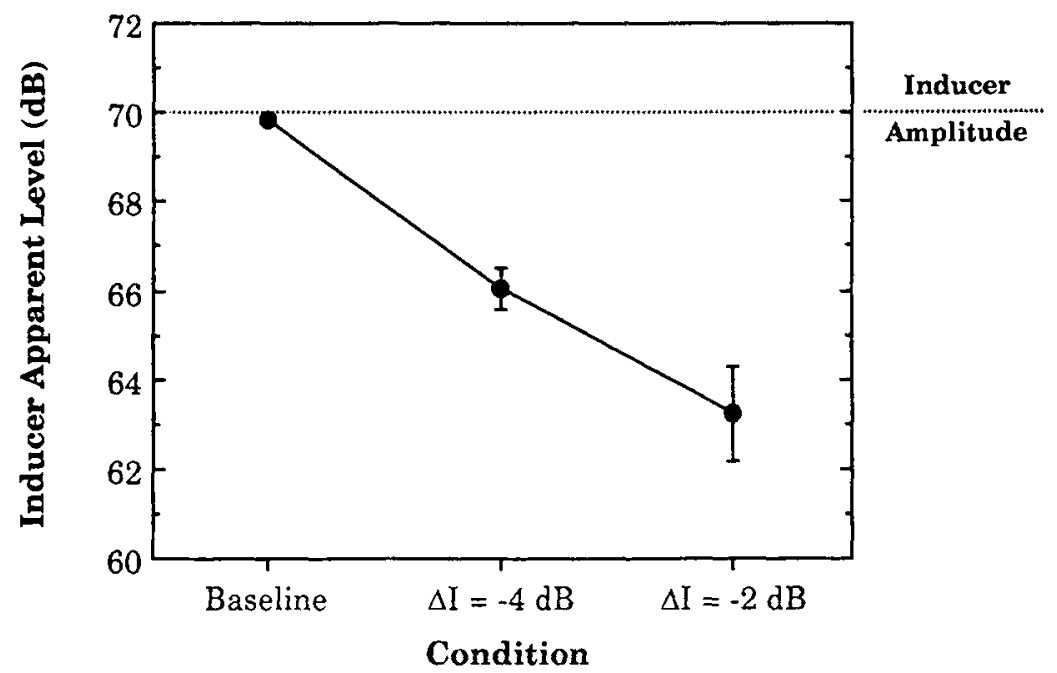

Figure 7. Auditory induction: Changes in the apparent level (loudness) of a 70-dB broadband noise inducer alternating with a broadband noise inducee that was lower in level by 4 or $2 \mathrm{~dB}$. The baseline condition represents loudness judgments obtained when the inducee was replaced by silence. Plotted values are means and standard errors for 4 listeners.

neme, and investigated whether or not listeners heard a change in the timbre or brightness (not the loudness) of the interpolated noise when it was used to restore the $/ \mathrm{s} /$. It was reported that two of his experiments demonstrated qualitative changes consistent with the application of auditory induction rules to phonemic restoration, whereas the other three experiments found no such change. Repp concluded that, overall, his results favored Bregman's position. Experiment 4 in the present study attempted to determine directly whether a loudness reduction occurs for narrowband noise interrupting narrowband speech, as predicted by induction theory, and whether the extent of such reduction follows the spectral dependency observed in Experiment 1 for tones.

\section{Method}

Stimuli. The verbal inducee used in this experiment was the sentence, "Where are you now?" The sentence, which was chosen for its relatively flat amplitude contour, was produced at an even level by a male speaker and had a duration of $1.25 \mathrm{sec}$. The initial recording was made digitally with a sampling frequency of $22 \mathrm{kHz}$ and 16-bit resolution. Amplitude measurements were made for successive 200 -msec segments by excising the segments and repeating them without pause. The first five segments had amplitudes matching within $\pm 2 \mathrm{dBA}$, and the last segment had a falling amplitude contour and was $8 \mathrm{dBA}$ below the average of the others (there was 50 -msec silence separating restatements of the sentence). This stimulus was played back in a looped format and subjected to $1 / 3$ octave bandpass filtering (Brüel \& Kjar Model 2121 frequency analyzer) with a center frequency set at $1.5 \mathrm{kHz}$. Sentences filtered in this fashion are fully intelligible and demonstrate phonemic restoration when interrupted by noise (Bashford et al., 1992; Bashford \& Warren, 1979, 1987). The same filtering apparatus was used for generating the five inducers, which were $1 / 3$-octave bands of Gaussian noise. One of the noise bands matched the $1.5-\mathrm{kHz}$ center frequency of the speech signal, and the remaining noise bands differed from this center frequency by $\pm^{1 / 2}$ octave and \pm 1 octave (i.e., 0.75 , $1.06,2.12$, and $3.0 \mathrm{kHz})$. The repeating narrowband sentence and the narrow bands of noise were recorded at $15 \mathrm{ips}$ on parallel tracks of an Otari Model MX 5050 eight-track recorder that was used for playback of stimuli during the experiment.

The filtered speech serving as the inducee and the filtered noises serving as inducers were passed through the same gating system employed in Experiment 3. On/off times were $125 \mathrm{msec}$, and the first stimulus for each method-of-limits trial consisted of 13 statements of the inducee alternating with 12 statements of the inducer, each sequence beginning and ending with bursts of the inducee (3.125-sec total duration). A 500-msec pause separated the induction stimulus from the comparison stimulus, which consisted of 4 bursts of the comparison noise alternating with 125 -msec silent gaps. The level of the comparison noise was controlled by the same computer-controlled amplifier used in Experiment 3. Throughout the experiment, the peak level of the repeating narrowband sentence was fixed at $70 \mathrm{~dB}$ SPL, and the average levels of the narrowband noise inducers were also fixed at $70 \mathrm{~dB}$ SPL. It should be noted that, in Experiments 1-3, the inducer frequency was fixed and the inducee frequency was varied, whereas in Experiment 4, in order to maintain speech intelligibility, the center frequency of the bandpassed sentence inducee was fixed but the center frequency of the bandpassed noise inducer was varied.

Procedure. The listeners provided 10 method-of-limits series of judgments -5 ascending series alternating with 5 descending seriesfor each of the five inducer noise bands within each of the four blocks. As in Experiment 1, the initial comparison levels for the ascending and descending series were varied pseudorandomly, and the successive comparison levels differed by $2 \mathrm{~dB}$. Also, as in Experiment 1 , the order of presentation for inducers was pseudorandomized within blocks, with the restriction that no inducer was presented successively across blocks. As in Experiment 3, each series of experimental trials for a given inducer was preceded by a series of baseline trials during which only the noise bursts (125-msec on/off time) were present during both trial intervals.

\section{Results and Discussion}

Figure 8 shows the change in apparent noise level, relative to baseline judgments with the speech absent, for each of the five inducer noise bands. The figure indicates that, in keeping with induction theory, loudness reduc- 


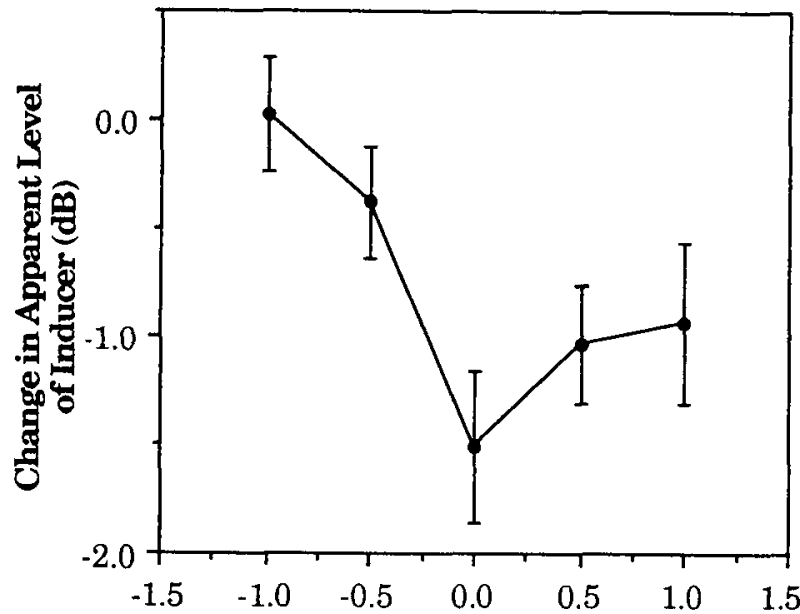

Inducee CF relative to Inducer CF (Octaves)

\begin{abstract}
Figure 8. Auditory induction of speech (phonemic restorations): Changes in the apparent level (loudness) of various noise-band inducers when interrupting an inducee consisting of a bandpass-filtered sentence. The $1 / 3$-octave speech band had a center frequency (CF) of $1.5 \mathrm{kHz}$ and was presented at a peak level of $70 \mathrm{~dB}$. The inducers were $1 / 3$-octave noise bands at $70 \mathrm{~dB}$ having CFs differing from the speech $\mathrm{CF}$ by $-1.0,-0.5,0.0,+0.5$ and +1.0 octaves. Plotted values are means and standard errors for 4 listeners.
\end{abstract}

tion did occur for narrowband noise interrupting narrowband speech, and that the same spectral dependency for tonal loudness reduction found in Experiment 1 applied to phonemic restoration as well. As with auditory induction of tones, loudness reduction of the inducer was an asymmetrical function of frequency separation, with a greater change occurring when the noise inducer had lower frequencies than did the speech inducee (inducee/inducer values of +0.5 and +1.0 , octaves, as shown in Figure 8), in keeping with the greater upward spread of neural activity along the basilar membrane produced by the inducer (see Warren et al., 1972). This spectral asymmetry was also observed when the limiting conditions for full restoration of narrowband speech by narrowband noise were determined (Bashford et al., 1992; Bashford \& Warren, 1979, 1987). ${ }^{3}$

The observations described above are supported by statistical analyses. The four listeners' mean level matches were subjected to a treatments $\times$ treatments $\times$ subjects ANOVA, which yielded a significant interaction $[F(4,12)$ $=12.46, p<.001]$ between the inducer condition (baseline with inducee absent vs. induction condition with inducee present) and the inducer-frequency condition. A subsequent simple-effects analysis indicated that the effect of inducer frequency was significant only for the induction condition $[F(4,12)=5.39, p<.025]$. A single$d f$ contrast that compared the mean for the two lowest frequency inducers with the mean for the two highest frequency inducers indicated that significantly greater loudness reduction occurred for the lower frequency inducers $[F(1,12)=14.28, p<.003]$, as would be predicted from induction theory. In addition, a comparison between the mean for the inducer noise band that matched the speech in center frequency (0.0-octave separation) and the combined mean for the noise bands $1 / 2$ octave above and below the speech center frequency indicated that the inducer matching the speech center frequency was subject to the greatest loudness reduction $[F(1,12)=27.17, p<.001]$.

A drop in loudness of interpolated noise conflicts with the hypothesis that phonemic restoration differs from restoration of nonverbal sounds in being "schemadriven," a process described as not involving the reallocation of auditory input (Bregman, 1990, p. 372). Perhaps it is possible to consider instead that all types of auditory induction are schema-driven in the sense that they are driven by contextually determined expectations. The schema employed can be as complex as those involved in speech perception or as simple as the expectation of continuity of an interrupted but otherwise steady single sound. In keeping with this view, all types of restoration follow the same basic rules of reciprocal interaction (including the reallocation of auditory input from inducer to inducee in accord with specific spectral dependencies).

\section{GENERAL DISCUSSION}

Our ability to perceptually restore segments of signals that have been obliterated or masked by extraneous sounds usually goes unnoticed, since the restored sounds seem as real as those physically present. Studies have demonstrated that perceptual restoration of obliterated sounds, or auditory induction, represents a quite sophisticated process-intact portions of the signal preceding and following the obliterated sound are used to identify the missing fragment. Auditory stimulation provided by the extraneous sound can then be used for the construction of the missing sound-by limiting the substrate for synthesis to the neural input provided by the extraneous sound, only those portions of the signal that could have been masked can be restored. Three types of restoration have been identified: (1) homophonic continuity, in which the restored sound (the inducee) and the potential masker (the inducer) are different amplitude levels of the same sound; (2) heterophonic continuity, in which the inducee and inducer differ in spectrum; and (3) contextual catenation, in which the restored inducee differs not only from the inducer but also from preceding and following portions of the signal (as in phonemic restorations; see Warren, 1984 , for a review of the literature and discussion of these different types of auditory induction). Studies of auditory induction have concentrated on the restoration of the inducee, generally ignoring the effects of induction on the inducer. The present study has examined changes occurring in the inducer for all three types of auditory induction.

It was observed in Experiment 1 that the homophonic induction of tones not only produced changes in the loudness of the inducer but also produced changes in its timbre. When two sinusoidal tones of the same frequency but different amplitudes were alternated every $200 \mathrm{msec}$, increasing the amplitude of the fainter tone caused the apparent level of the higher amplitude tone to decrease (it 
eventually appeared to be fainter than the lower amplitude inducee), and, in addition, the quality of the inducer changed so that it no longer appeared tonal. In Experiment 3, it was found that homophonic induction of a broadband noise also involved a reduction in loudness of the higher amplitude inducer. However, the change in inducer quality was less dramatic for noise. Informal observations have indicated that auditory induction is heard for noise when the difference in level of the inducer and inducee is decreased to $1 \mathrm{~dB}$ or even less. An intriguing problem is how induction could occur when long-term rms levels of a broadband noise differing by as little as $0.5 \mathrm{~dB}$ were alternated every $200 \mathrm{msec}$, given the random fluctuations in amplitude within critical bands of this stochastic signal.

In the case of tones differing in both amplitude and frequency, partial heterophonic induction occurred-the apparent duration of the fainter tonal inducee increased (Experiment 2) as the apparent amplitude of the inducer decreased (Experiment 1). Thus, heterophonic auditory induction of the fainter of two alternating sounds is not an all-or-none phenomenon taking place when threshold conditions are reached-it rather represents a continuum of interaction between inducee and inducer, having as a limit the complete closure of the gaps separating successive statements of the inducee. It appears that an increase in the apparent duration of the inducee is achieved by allocating to the inducee a portion of the auditory input corresponding to the inducer, which results in a decrease in the apparent amplitude of the inducer. In addition to this loudness decrease, a change in the quality of the inducer occurs, which can be attributed to the anomalous auditory representation of the inducer following the transfer of particular portions of its neural representation required for the perceptual synthesis of the inducee.

Thus, auditory induction involves a reciprocal interaction between inducer and inducee-the increase in apparent duration of the inducee is accompanied by a decrease in the apparent amplitude of the inducer. Although it has been suggested that phonemic restoration differs from nonverbal restoration, it was found that loudness reduction occurred also for noise interrupting speech, indicating that reallocation of the inducer's neural input mediates the synthesis of missing verbal fragments, as it does with other forms of auditory induction.

Finally, it should be noted that auditory induction in its various forms represents a highly sophisticated mechanism capable of reversing the effects of masking, which becomes fully activated only when the characteristics of an interrupting noise would permit obliteration of the signal were it actually present.

\section{REFERENCES}

BASHFord, J. A., JR., RienER, K. R., \& WARREN, R. M. (1992). Increasing the intelligibility of speech through multiple phonemic restorations. Perception \& Psychophysics, 51, 211-217.
Bashford, J. A., JR., \& WARRen, R. M. (1979). Perceptual synthesis of deleted phonemes. In J. J. Wolf \& D. H. Klatt (Eds.), Speech communication papers (pp. 423-426). New York: Acoustical Society of America.

Bashford, J. A., JR., \& Warken, R. M. (1987). Multiple phonemic restorations follow the rules for auditory induction. Perception \& Psychophysics, 42, 114-121.

Bregman, A. S. (1990). Auditory scene analysis. Cambridge, MA: MIT Press.

Houtgast, T. (1972). Psychophysical evidence for lateral inhibition in hearing. Journal of the Acoustical Society of America, 51, 1885-1894.

JAVEL, E. (1986). Basic response properties of auditory nerve fibers. In R. S. Altschuler, D. W. Hoffman, \& R. P. Bobbin (Eds.), Neurobiology of hearing: The cochlea (pp. 213-245). New York: Raven Press.

NETER, J., \& WASSERMAN, W. (1974). Applied linear statistical models. Homewood, IL: Richard D. Irwin.

REPP, B. H. (1992). Perceptual restoration of a "missing" speech sound: Auditory induction or illusion? Perception \& Psychophysics, 51, 14-32.

SMITH, R. L. (1988). Encoding of sound intensity by auditory neurons. In G. M. Edelman, W. E. Gall, \& W. M. Cowan (Eds.), Auditory function: Neurobiological bases of hearing (pp. 243-274). New York: Wiley.

WARREN, R. M. (1984). Perceptual restoration of obliterated sounds. Psychological Bulletin, 96, 371-383.

W ArRen, R. M., Obusek, C. J., Ackroff, J. M. (1972). Auditory induction: Perceptual synthesis of absent sounds. Science, 176, 1149-1151.

Warren, R. M., \& Sherman, G. L. (1974). Phonemic testorations based on subsequent context. Perception \& Psychophysics, 16, 150-156.

Wrightson, J. M., \& WARREN, R. M. (1981). Incomplete auditory induction of tones alternated with noise: Effects occurring below the pulsation threshold. Journal of the Acoustical Society of America, 69, S105-S106. (Abstract)

\section{NOTES}

1. During the 10-msec switching period, the phase relations between the inducer and the inducee were constantly changing for all stimuli except for the homophonic ( $1-\mathrm{kHz}$ inducee) condition. To determine whether the fixed phase relations during the $10-\mathrm{msec}$ rise/fall segment of the homophonic stimuli could have an effect on induction, we examined the two limiting phase relations of $0^{\circ}$ and $180^{\circ}$. The same four listeners serving in Experiment $1 \mathrm{~A}$ used the method of adjustment, and each provided three matches to the inducer level when the inducer and inducee were in-phase $\left(0^{\circ}\right)$ and three matches when they were out-ofphase $\left(180^{\circ}\right)$ relative to each other. All listeners reported hearing a continuous tone and a pulsing tone for both phase conditions, and there was a nonsignificant difference $[F(1,3)=3.41, p>.15]$ between the $0^{\circ}$ and $180^{\circ}$ conditions in the adjusted level of the comparison tone.

2. A supplemental experiment was also conducted to measure possible effects of homophonic induction on the apparent level of the inducee. Our four listeners provided six loudness matches, using the method of adjustment, for a $1-\mathrm{kHz}, 66-\mathrm{dB}$ inducee as it alternated with a $1-\mathrm{kHz}$, 70- $\mathrm{dB}$ inducer, under the same switching conditions employed in our formal experiments. Across listeners, the level match averaged $66.2 \mathrm{~dB}$, with a standard error of $0.30 \mathrm{~dB}$, indicating that the inducee's level was perceived veridically under the conditions of the present study.

3. While these earlier studies did not relate the restoration of speech to changes occurring in the inducing noises, listeners in the present study reported that phonemic restoration (i.e., full continuity) occurred only with the two noisebands that were found to undergo the greatest reduction in apparent level.

(Manuscript received April 30, 1993; revision accepted for publication August 3, 1993.) 